\title{
Determinants of Abnormal Liver Function Tests in Diabetes Patients in Myanmar
}

\author{
Han $\mathrm{Ni}^{1, *}$, Htoo Htoo Kyaw Soe ${ }^{2}$, Aung Htet ${ }^{3}$ \\ ${ }^{1}$ Department of Internal Medicine, Melaka Manipal Medical College, Melaka, 75150, Malaysia \\ ${ }^{2}$ Department Community Medicine, Melaka Manipal Medical College, Melaka, 75150, Malaysia \\ ${ }^{3}$ Department of Diagnostic Radiology, Sin gapore General Hospital, 169608, Singap ore
}

\begin{abstract}
Abnormal liver function tests are not uncommon encounter in diabetes mellitus patients. The aim of this study was to find out the liver function test abnormalities in a group of diabetic patients in Myanmar and to determine the factors associated with these biochemical changes. In this cross sectional study conducted at the diabetic clinic of Yangon General Hospital, Myanmar between March 2006 and February 2007, a total of 81 patients were included. The means of alanine aminotransferase (ALT), aspartate aminotransferase (AST), alkaline phosphatase (ALP), $\gamma$ glutamyl transferase $(\gamma$ GT), bilirubin and prothrombin time fall within normal range. Raised ALT and AST were noted in $18.5 \%$ and $14.8 \%$ respectively. $4.9 \%$ had high bilirubin and prolonged prothrombin time each, $\gamma$ GT was normal in all patients. The mean values of ALT and AST had no significant correlation with age, fa mily history of diabetes, mode of therapy or type of diabetes. Although mean ALT had no correlation with gender of the diabetes patients, AST was found to be significantly elevated in men. Values of ALT and AST were also significantly higher with increasing BMI. Hepatomegaly, increased liver echogenicity and presence of fatty liver were the factors significantly correlated with elevated ALT and AST values. In conclusion, abnormal liver function results are more common among diabetes patients. Elevated ALT and AST are the markers for associated non alcoholic fatty liver disease in diabetes patients. Testing for ALT and AST, should be carried out to screen underlying fatty liver, especially in male diabetes patients with high BMI.
\end{abstract}

Keywo rds Liver Function Tests, Diabtes Mellitus, Myanmar, Alanine A minotransferase, Aspartate Aminotransferase

\section{Introduction}

Diabetes mellitus is one of the major non-communicable diseases and the prevalence is rising globally. Type 2 diabetes is the most common form, accounting for $90 \%$ of all cases[1]. The prevalence of diabetes worldwide was estimated to be $2.8 \%$ in 2000 and $4.4 \%$ in 2030 . The total number of diabetes is projected to increase from 171 million in 2000 to 366 million in 2030. Diabetes is more prevalent in men than wo men[2].

There exists an association between diabetes and liver injury. Liver plays a major role in the regulation of carbohydrate homeostas is. Hepatocellular glycogen accumulation leads to hepatomegaly and liver enzyme abnormalities in poorly controlled diabetes patients. In hyperglycemic states, there will be intracellu lar glycogen accumulation in the hepatocytes due to increased glycogen synthesis, causing typical biochemical findings of mild to moderately elevated aminotransferases, normal liver

* Corresponding author:

hanni.dr@gmail.com (Han Ni)

Published online at http://journal.sapub.org/diabetes

Copyright (C) 2012 Scientific \& Academic Publishing. All Rights Reserved synthetic function, with or without mild elevations of alka line phosphatase. All these biochemical disturbances and hepatomegaly are found to be reversible with good glycemic control[3].

Secondly, liver can be affected by steatosis or accumulation of fat, a condition known as non-alcoholic fatty liver disease (NAFLD). It is a well-recognized complication of diabetes with frequency of 40-70\%[4]. Associated obesity is a confounding variable for fatty liver. Increased transport of fatty acids to the liver, enhanced hepatic fat synthesis as well as decreased oxidation or removal of fat from the liver lead to fat accumulation in the liver. The steatosis is either microvesicular or macrovesicular and is found to progress to fibrosis and cirrhosis. The most common clinical finding is hepatomegaly, with normal or only mildly elevated transaminases and normal bilirubin. These changes are not reversible with sustained glucose control[4].

Nonalcoholic fatty liver disease (NAFLD) is the main cause of chronic liver disease associated with diabetes and obesity. It was first reported in 1980's in obese females with diabetes. Without treatment, compensated steatosis in NAFLD will eventually lead to decompensated steatosis with necroinflammation and fibrosis, i.e stage of 
non-alcoholic steatohepatitis (NASH). NASH is a leading cause of end-stage liver disease and also a contributor of cardiovascular disease in type 2 diabetes mellitus [5].

Definitive diagnosis of NASH requires liver biopsy. However, new imaging methods like transient elastography and plasma biomarkers such as cytokeratin-18 fragments (marker of hepatocyte apoptosis) are emerging as alternative diagnostic techniques. Lifestyle modification is the gold standard in the management of NASH. Thiazolidiendiones have shown some promising results in recent randomized controlled trials [5].

Serum amino transferases such as alanine aminotransferase (ALT) and aspartate aminotransferase (AST) indicate the concentration of hepatic intracellular enzy mes that have leaked into the circulation. These are the markers for hepatocellular injury and are used as primary screening of NASH[6].

Chronic mild elevations of ALT and AST are seen in type 2 diabetes patients. In a study done in United States by Erbey et al in 2000, the prevalence of elevated ALT among type 2 diabetes is $7.8 \%$ compared to $3.8 \%$ in those without diabetes[7]. In another study by Salmela et al, elevated ALT in diabetes patients was associated with increased BMI and poor glycemic control in multivariate analys is [8].

Elevated liver enzy mes are found to be pred ictors of future cardiovascular disease in some studies. In the Firen ze Bagno a Ripoli (FIBAR) study done in Italy, raised gamma-GT of more than $40 \mathrm{U} / \mathrm{L}$ and AST of more than $40 \mathrm{U} / \mathrm{L}$ were associated with increased incidence of Cardiovascular Disease (CVD)[9]. However, subsequent reviews conclude that diagnosis of NAFLD is insufficient to consider as high risk for CVD. The presence of NAFLD should prompt for screening of diabetes, but CVD screening should be guided by other established cardiovascular risk factors [10].

In Myanmar, the estimated prevalence of diabetes mellitus was $2.4 \%$ in $1995,2.5 \%$ in 2000 and expected to be $3.2 \%$ in 2025 according to World Health Organization (WHO) estimates and projections 1995-2025[11]. There is no doubt that the morbidity of diabetes mellitus is increasing in Myanmar, especially in the urban population.

The aim of this current study was to evaluate the liver function among diabetes patients in Myanmar and to find out the factors as sociated with these abnormalities.

\section{Materials and Methods}

\subsection{Study design and Subjects}

This study was a hospital based cross sectional descriptive study conducted at the diabetic clinic of Yangon General Hospital, Yangon, Myanmar between March 2006 and February 2007. Subjects were recruited according to simple random sampling method meeting the selection criteria.

\subsection{Selection Criteria}

\subsubsection{Inclusion Criteria}

The patients with confirmed diabetes mellitus or newly diagnosed diabetes mellitus by WHO criteria (1999), fasting plasma venous glucose of $\geq 7 \mathrm{mmol} / 1(126 \mathrm{mg} / \mathrm{dl})$ or random or two hour post prandial plas ma venous glucose of $\geq 11.1$ $\mathrm{mmol} / 1(200 \mathrm{mg} / \mathrm{dl})$.

\subsubsection{Exclusion Criteria}

The diabetic patients with history of alcohol intake, hepatotoxic drugs like amiodarone, antituberculous drugs, history of liver diseases or clinical evidence of acute hepatitis, those who were found to have evidence of hepatitis $\mathrm{B}$ and $\mathrm{C}$ virus infection (HBs Ag positive and HCV antibody positive) were excluded from this study.

\subsection{Ethical Consider ations}

The Institution's Ethical Committee approval was obtained prior to the enrolment of subjects. The objectives and the detailed procedures of blood taking and imaging involved in the study were explained to all eligible subjects for this study. Emphasis was given that participation in this study was voluntary. Written consent forms were obtained fro mall partic ipants who agreed to take part in the study.

\subsection{Questionnaire and Biodata Collection}

A questionnaire was specifically designed to obtain information which helps to select individuals according to the selection criteria of the study. The questions also focused on sociodemographic data (age, sex, ethnicity) and background characteristics of diabetes (family history of diabetes, duration and type of diabetes mellitus, mode of anti-diabetic therapy).

Weight and height were measured using standard procedures and Body Mass Index (BMI) was then calculated fro $m$ the formu la of weight $(\mathrm{kg}) /$ height $\left(\mathrm{m}^{2}\right)$.

\subsection{Blood Sample}

$5 \mathrm{ml}$ of venous blood was drawn from each volunteer in this study using a disposable plastic syringe. The sample was then analysed for serum bilirubin, alanine a minotransferase (ALT), aspartate aminotransferase (AST), alkaline phosphatase (ALP), $\gamma$ glutamyl transferase (GGT), prothrombin time (PT) by 550 Expressed Plus Automatic Chemistry Analyzer at the pathology department of Yangon General Hospital.

\subsection{Imaging of Liver}

Ultrasonographic examination of abdomen was carried out at the radiology department of Yangon General Hospital to determine the size, echogenicity of liver and the presence of fatty liver.

\subsection{Statistical Analysis}

The data collected during the current study were recorded and analyzed statistically to determine the significance of different parameters by using SPSS package for windows version 16.0. 


\section{Results}

Table 1. Demographic and clinical characterist ics of diabetes patients $(n=$ 81)

\begin{tabular}{|c|c|}
\hline Variable & $\mathrm{N}(\%)$ \\
\hline \multicolumn{2}{|l|}{ Age } \\
\hline$\leq 55$ & $33(40.7)$ \\
\hline$>55$ & $48(59.3)$ \\
\hline \multicolumn{2}{|l|}{ Ethnicity } \\
\hline Bamar (Myanmar) & $71(87.7)$ \\
\hline Indian & $8(9.9)$ \\
\hline Others & $2(2.5)$ \\
\hline \multicolumn{2}{|l|}{ Gen der } \\
\hline Male & $23(32.1)$ \\
\hline Female & $55(67.9)$ \\
\hline BMI & $25.84 \pm 1.81$ \\
\hline \multicolumn{2}{|l|}{ Type of DM } \\
\hline Type 1 & $2(2.5)$ \\
\hline Type 2 & $79(97.5)$ \\
\hline \multicolumn{2}{|l|}{ Treatment } \\
\hline Insulin & $8(9.9)$ \\
\hline OHA & $73(90.1)$ \\
\hline \multicolumn{2}{|l|}{ Family history of DM } \\
\hline Absent & $30(37.0)$ \\
\hline Present & $51(63.0)$ \\
\hline \multicolumn{2}{|l|}{ Liver Size } \\
\hline Normal & $66(81.5)$ \\
\hline Increased & $15(18.5)$ \\
\hline \multicolumn{2}{|l|}{ Liver echogenicity } \\
\hline Normal & $62(76.5)$ \\
\hline Increased & $19(23.5)$ \\
\hline \multicolumn{2}{|l|}{ Fatty Liver } \\
\hline Absent & $68(84.0)$ \\
\hline Present & $13(16.0)$ \\
\hline
\end{tabular}

A total of 81 confirmed diabetes patients participated in this study. Table 1 shows demographic and clinical characteristics. Mean age was $56.99 \pm 9.06$ years and ranged from 22 years to 75 years. Majority of the patients were Bamar $(87.7 \%)$ and female represented $67.9 \%$. Mean BMI was 25.84 , ranging from 22.5 to 30.5 . Most of them $(97.5 \%)$ had type 2 diabetes while only 2 patients $(2.5 \%)$ had type 1 diabetes. $90.1 \%$ were treated with $\mathrm{OHA}$ and $63 \%$ had family history of diabetes. Regarding clinical characteristics, only $18.5 \%$ of the patients had increased liver size, $23.5 \%$ had increased liver echo and $16 \%$ had fatty liver. (Table 1)

Mean value of ALT and AST were 42.94 and 29.69 respectively. Moreover, in th is study, serum bilirubin, ALP, rGT and PT were estimated and mean values were 9.13, 102.04, 23.21 and 11.51 respectively. (Table 2).

Table 2. Mean value of biochemical parameters among diabet es patients $(\mathrm{n}=81)$

\begin{tabular}{cccc}
\hline Variable & $\begin{array}{c}\text { Mean } \pm \\
\text { SD }\end{array}$ & $\begin{array}{c}\text { Laboratory } \\
\text { reference } \\
\text { range }\end{array}$ & $\begin{array}{c}\text { No of patients } \\
\text { outsidethe } \\
\text { reference } \\
\text { range }(\%)\end{array}$ \\
\hline ALT & $\begin{array}{c}42.94 \pm \\
20.12\end{array}$ & $\begin{array}{c}21-72(\mathrm{M}) \\
9-52(\mathrm{~F})\end{array}$ & $15(18.5 \%)$ \\
AST & $29.69 \pm$ & $17-59(\mathrm{M})$ & $12(14.8 \%)$ \\
Serum & 11.73 & $14-36(\mathrm{~F})$ & \\
bilirubin & $7.13 \pm$ & $2-20$ & $4(4.9 \%)$ \\
ALP & $102.04 \pm$ & $38-126$ & $5(6.2 \%)$ \\
rGT & 22.82 & & 0 \\
& $23.21 \pm$ & $7-47$ & $4(4.9 \%)$ \\
\hline
\end{tabular}

M: Male, F: Female

Table 3 showed the relationship between demographic, clinical characteristics and liver function test (AST, ALT). Age was divided into two groups, less than and equal to 55 years and more than 55 years; no significant difference was noted in the means of ALT and AST between these two groups. Likewise, there was no significant difference in mean of ALT between male and female. However, AST was significantly found to be higher in male. Means of ALT and AST also not significantly diffe red between type 1 and type 2 diabetes, different types of treatment, family history of diabetes. The mean values of ALT and AST were significantly higher among patients with increased liver size, presence of fatty liver and increased liver echo. (Table 3)

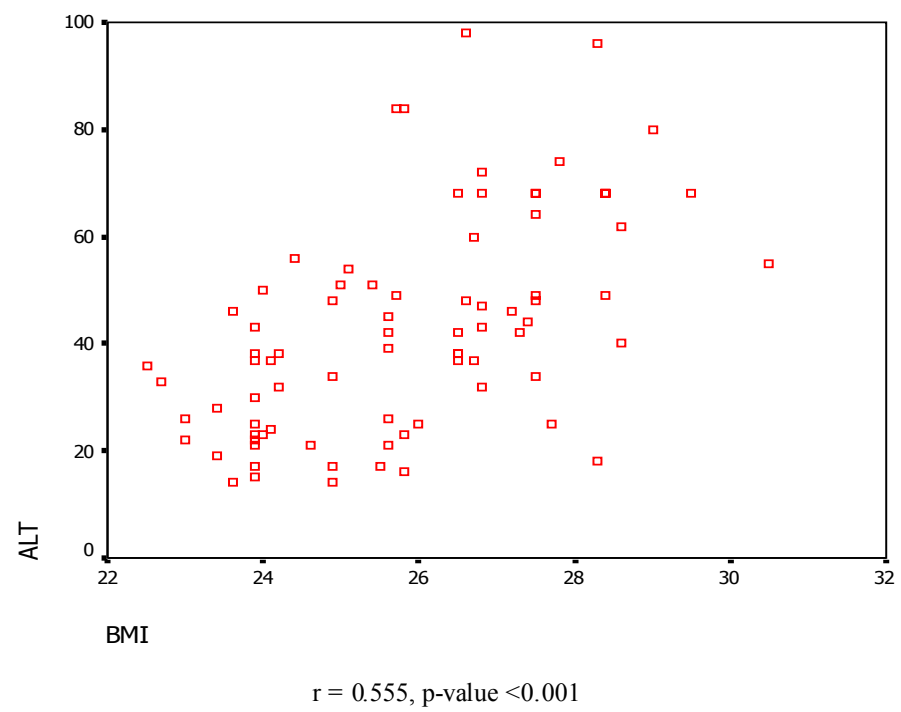

Figure 1. Correlation between ALT and BMI among diabet es patients $(n=81)$ 
Table 3. Relationship between demographic, clinical characteristics and liver function tests (AST, ALT) among diabetes patients $(\mathrm{n}=81$ )

\begin{tabular}{|c|c|c|c|c|c|c|c|c|}
\hline \multirow{2}{*}{ Variable } & \multicolumn{4}{|c|}{ ALT } & \multicolumn{4}{|c|}{ AST } \\
\hline & Mean \pm SD & $\mathrm{t}$ & $95 \% \mathrm{CI}$ & $\mathrm{p}$-value & Mean \pm SD & $\mathrm{t}$ & $95 \% \mathrm{CI}$ & p-value \\
\hline \multicolumn{9}{|l|}{ Age } \\
\hline$\leq 55$ & $42.21 \pm 20.15$ & -0.27 & $-10.33-7.88$ & 0.790 & $30.97 \pm 13.03$ & 0.81 & $-3.14-7.45$ & 0.420 \\
\hline$>55$ & $43.44 \pm 20.09$ & & & & $28.81 \pm 10.81$ & & & \\
\hline \multicolumn{9}{|l|}{ Gen der } \\
\hline Male & $47.58 \pm 23.19$ & 1.44 & $-2.64-16.29$ & 0.155 & $34.23 \pm 14.95$ & 2.09 & $0.21-13.16$ & $0.043^{*}$ \\
\hline Female & $40.74 \pm 18.31$ & & & & $27.54 \pm 9.27$ & & & \\
\hline \multicolumn{9}{|c|}{ Type of DM } \\
\hline Type 1 & $22.0 \pm 1.41$ & -1.50 & $-49.92-6.98$ & 0.137 & $23.0 \pm 7.07$ & -0.81 & $\begin{array}{c}-23.62- \\
9.89\end{array}$ & 0.418 \\
\hline Type 2 & $43.47 \pm 20.09$ & & & & $29.86 \pm 11.81$ & & & \\
\hline \multicolumn{9}{|c|}{ Tre atment } \\
\hline Insulin & $44.87 \pm 20.44$ & 0.28 & $\begin{array}{c}-12.85- \\
17.15\end{array}$ & 0.776 & $28.50 \pm 10.69$ & -0.30 & $\begin{array}{c}-10.07- \\
7.43\end{array}$ & 0.764 \\
\hline OHA & $42.73 \pm 20.21$ & & & & $29.82 \pm 11.91$ & & & \\
\hline \multicolumn{9}{|c|}{ Family history } \\
\hline Absent & $42.53 \pm 21.39$ & -0.14 & $-9.91-8.62$ & 0.891 & $29.73 \pm 12.70$ & 0.02 & $-5.34-5.47$ & 0.980 \\
\hline Present & $43.18 \pm 19.54$ & & & & $29.67 \pm 11.26$ & & & \\
\hline \multicolumn{9}{|c|}{ Liver size } \\
\hline Normal & $38.03 \pm 17.96$ & -5.34 & $\begin{array}{c}-36.38- \\
-16.62\end{array}$ & $0.000^{*}$ & $27.88 \pm 10.92$ & -3.06 & $\begin{array}{c}-16.14- \\
-2.61\end{array}$ & $0.010^{*}$ \\
\hline \multicolumn{8}{|l|}{ Fatty liver } & \\
\hline Absent & $38.18 \pm 17.74$ & -5.77 & $\begin{array}{c}-39.89- \\
-19.44\end{array}$ & $0.000^{*}$ & $27.81 \pm 10.84$ & -3.53 & $\begin{array}{c}-18.34- \\
-5.12\end{array}$ & $0.001^{*}$ \\
\hline Present & $67.85 \pm 11.87$ & & & & $39.54 \pm 3.25$ & & & \\
\hline \multicolumn{9}{|c|}{ Liver echo } \\
\hline Normal & $36.16 \pm 16.16$ & -6.88 & $\begin{array}{c}-37.24- \\
-20.54\end{array}$ & $0.000^{*}$ & $26.52 \pm 8.86$ & -3.96 & $\begin{array}{c}-20.62- \\
-6.45\end{array}$ & $0.001^{*}$ \\
\hline Increased & $65.05 \pm 15.49$ & & & & $40.05 \pm 14.08$ & & & \\
\hline
\end{tabular}

*significant

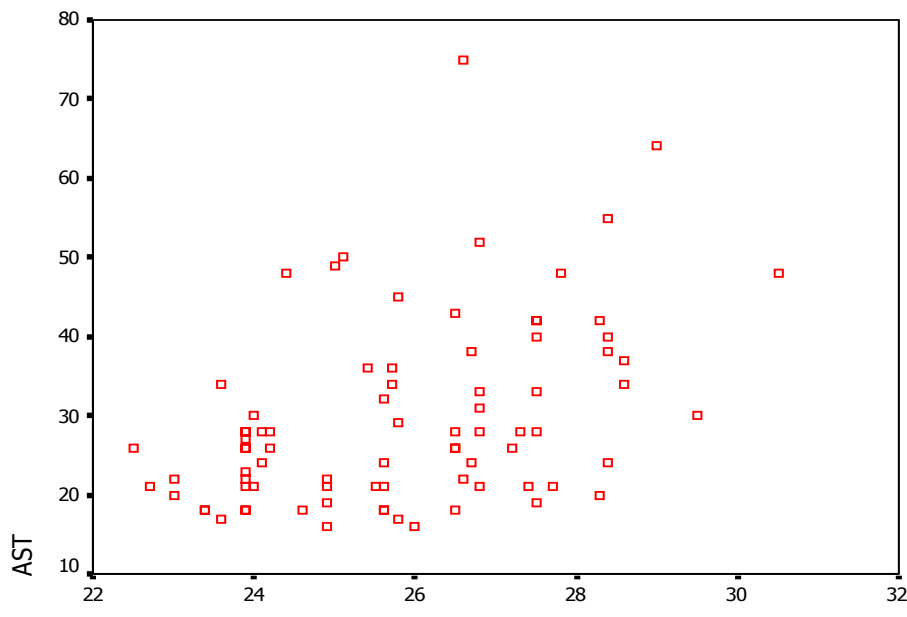

BMI

$\mathrm{r}=0.431, \mathrm{p}$-value $<0.001$

Figure 2. Correlation between AST and BMI among diabetes patients $(\mathrm{n}=81)$

Figure 1 and 2 show the correlation between ALT, AST and BMI among 81 diabetes patients. There was significant positive correlation between ALT and BMI $(r=0.555$, p-value $<0.001)$ as ALT increased with increasing BMI (Figure 1). Similarly, AST significantly increased with increase in BMI showing significant positive correlation $(\mathrm{r}=$ 0.431 , p-value $<0.001$ ) (figure 2).

\section{Discussions}


Type 2 diabetes patients have been reported to be associated with higher incidence of abnormal liver function tests (LFT) compared to the individuals without diabetes, elevated ALT being the most common abnormality[12].

Salmela et al in 1984 studied the liver function tests of 175 diabetic patients without chronic liver disease, where $57 \%$ were found to have at least one abnormal LFT, $27 \%$ had at least two abnormal LFTs. However, these increases in liver function values were rarely more than two times of the upper limit of normal[8].

A cross sectional study from Iran demonstrated a rise of ALT and AST in $10.4 \%$ and $3.3 \%$ of type 2 diabetes patients respectively[6].

In a UK cohort study of 959 diabetic patients over four year period, $15.7 \%$ had raised ALT, $10.4 \%$ had elevated alkaline phosphatase whereas only $3.9 \%$ had hyperbilirubin aemia[13]. Another study of 60 well controlled diabetic outpatients showed elevation of alkaline phosphatase and $\gamma$ GT in 11 and 10 patients respectively, but the rise was not more than two times upper limit of normal value[14].

According to a study in Sudan, where 50 diabetes patients and 30 normal control subjects were tested for liver function, the means of ALT, AST, $\gamma \mathrm{GT}$, total protein and albumin were reported to be significantly higher among diabetes compared to the control. However, the mean values were within the normal range[15]. In this study, $22 \%$ had at least one abnormal liver function test.

Similar finding was also present in a study by Foster et al, in which the means of ALT, AST, ALP, $\gamma$ GT, bilirubin and albumin of 60 study subjects with diabetes were within the reference range[14].

Likewise in our study, the means of ALT, AST, ALP, $\gamma \mathrm{GT}$, bilirubin and prothrombin time were within normal range among 81 diabetes patients. Raised ALT and AST were noted in $18.5 \%$ and $14.8 \%$ respectively. $4.9 \%$ had high bilirubin and prolonged prothromb in time. In contrast to the study by Salmela et al, where raised $\gamma \mathrm{GT}$ was reported in $19 \%$ of diabetes patients [8], none of the diabetes patients in our study had elevated $\gamma \mathrm{GT}$.

Abnormal liver function tests in diabetes patients can be attributed to several factors, the most common cause being non alcoholic fatty liver disease (NAFLD) associated with insulin resistance and metabolic syndrome in diabetes. Recently, studies have revealed the association of hepatitis $\mathrm{C}$ virus infection in diabetes patients causing deranged LFTs[16]. Though relatively infrequent, statin therapy can also contribute to abnormal liver function results [12].

Majority of the studies on liver function in diabetes excluded the hepatotoxic causes of viral hepatits, alcohol and medications. These studies focused on the factors influencing the liver function in diabetes.

Elevated ALT was significantly associated with overweight, short duration of diabetes, poor glycemic control and those treated with diet control or oral agents on multivariate analys is according to Salmela et al. In this study, the histologic changes were also studied but no significant correlation was present between liver histology and raised ALT nor $\gamma \mathrm{GT}[8]$.

Contrary to the above study, Foster et al reported no significant correlation between measures of glucose control and abnormal liver function tests. However, they found that presence of abnormally bright liver echo on ultrasound imaging was associated with minimally elevated ALT and ALP. This bright liver echo was due to fatty in filtration of liver[14].

Among the Iran diabetes population, although raised ALT was seen with increasing age, fasting blood glucose and triglyceride levels, it was not statistically significant. The risk of higher ALT was significantly lower with longer duration of diabetes. The incidence of high ALT was more common among men[6].

Erbey JR et al reported that the prevalence of elevated ALT levels among the U.S. type 2 diabetics was $7.8 \%$ and this prevalence was higher among obese $\left(\mathrm{BMI}>25 \mathrm{~kg} / \mathrm{m}^{2}\right)$ than non-obese diabetics (10.6\% vs. 6.6\%)[7].

In Sudan, high serum alanine aminotransferase level is greater among type 2 diabetes, overweight or obese and men[15].

The elevated ALT levels were significantly related to a BMI of $>25 \mathrm{~kg} / \mathrm{m}^{2}$ among the type 2 diabetics $(p=0.0103)$ in a study conducted in India. Positive correlation was also reported between glycaemic control (FBS and PPBS) and duration of diabetes mellitus with the ALT levels in this Indian study[17].

According to a study in India, age, duration of diabetes, presence of hypertension, family history of diabetes mellitus, body mass index, waist circumference, treatment with Metformin, and prevalence of metabolic syndrome were similar between diabetes patients with and without Non Alcoholic Steatohepatitis (NASH)[18].

Similarly, in our study, no significant correlation was noted between means of ALT and AST with age, family history of diabetes, mode of therapy or type of diabetes. Mean ALT had no correlation with sex of the population. However, AST was found to be significantly correlated with male sex. Values of ALT and AST were also significantly higher with increasing BMI.

A large cohort study done in India reported the presence of fatty infiltration of liver in ultrasonography in 62.25\% (127 out of 204 diabetes patients)[18]. Our study revealed presence of fatty liver in $16 \%$ of diabetes patients on ultrasound scan.

Raised liver en zy mes can be a marker of steatohepatitis in diabetes patients. Nevertheless, evidence fails to show significant positive correlation between liver histology and biochemical derangement 8 , 18]. Advanced degree of $\mathrm{NASH}$ and fibrosis can occur in diabetic patients without significant liver function abnorma lities [18].

In the present study, means of ALT and AST were significantly correlated with hepatomegaly, increased liver echo and presence of fatty liver on ultrasound imag ing. This might be due to the presence of non alcoholic steatohepatits 
in these patients. However, exact confirmation will require histological examination which is invasive.

Larger studies are recommended in future to find out the exact association between the biochemical and histological changes of liver in diabetes patients without chronic liver pathology in Myanmar.

\section{Conclusions}

Based on the findings of this study, raised ALT and AST are more common among the diabetes patients with higher BMI. AST elevation was also significant among men. Derangement of liver enzymes correlated statistically significantly with fatty liver on ultrasound. Therefore, abnormal liver function tests among diabetes patients can be indicator of associated non alcoholic fatty liver disease. Thus, checking for liver enzy mes, ALT and AST, should be carried out to screen the possibility of underlying fatty liver, which might need further evaluation and early intervention to prevent from progression into cirrhosis and chronic liver disease, especially in male patients with high BMI.

\section{ACKNOWLEDGEMENTS}

We are much grateful to all the patients who had voluntarily participated in this study as well as the staffs from the Pathology and Radiology department of Yangon General Hospital for their utmost contribution in completing this study. Our special thanks go to Professor Nyunt Thein, Professor Myint Han, Professor Tint Swe Latt and Professor Khin Maung Win from Yangon General Hospital for their encouragement, continuous support and guidance in undertaking this work.

\section{REFERENCES}

[1] Amos AF, McCarty DJ, Zimmet P. The rising global burden of diabetes and its complications: estimates and projections to the year 2010. Diabetes Med 1997; 14(5): 81-85.

[2] Wild S, Roglic G, Green A, Sicree R, King H. Global Prevalence of Diabetes: Estimates for the year 2000 and projections for 2030. Diabetes Care 2004; 27(5):1047-1053.

[3] Chatila R, West AB. Hepatomegaly and abnormal liver tests due to gly cogenosis in adults with diabetes. Medicine 1996; 75(6):327-33.

[4] Levinthal G.N, Tavill A.S. Liver disease and diabetes mellitus. Clin Diabetes 1999; 17 (2): 1-20.
[5] Kenneth Cusi. Nonalcoholic fatty liver disease in type 2 diabetes mellitus. Current Opinion in Endocrinology, Diabetes \& Obesity 2009; 16:141-149.

[6] Meybodi M A, Afkhami-Ardekani M, Rashidi M. Prevalence of Abnormal Serum Alanine Aminotransferase Levels in Type 2 Diabetic Patients in Iran. Pakistan Journal of Biological Sciences 2008; 11:2274-2277.

[7] Erbey JR, Silberman C, Lydick E. Prevalence of abnormal serum alanine aminotransferase levels in obese patients and patients with type 2 diabetes. Am. J. Med. 2000; 109: 588-590.

[8] Salmela PI, Sotaniemi EA, Niemi M, Maentausta O. Liver function tests in diabetic patients. Diabetes Care 1984; 7: $248-254$.

[9] Monami M, Bardini G, Lamanna C, Pala L, Cresci B, Francesconi $\mathrm{P}$, et al. Liver enzymes and risk of diabetes and cardiovascular disease: results of the Firenze Bagno a Ripoli (FIBAR) study. Metabolism 2008; 57(3):387-92.

[10] Ghouri N, Preiss D, Sattar N. Liver enzymes, nonalcoholic fatty liver disease, and incident cardiovascular disease: a narrative review and clinical perspective of prospective data. Hepatology 2010; 52(3):1156-61.

[11] World Health Organization, Headquarters. Division of Non-communicable Diseases, Diabetes.

[12] Harris E H. Elevated Liver Function Tests in Type 2 Diabetes. Clinical Diabetes 2005; 23 (3): 115-119.

[13] Sherif Gonem, Alan Wall, Parijat De. Prevalence of abnormal liver function tests in patients with diabetes mellitus Endocrine Abstracts 2007; 13:157.

[14] Foster K J, Dewbury K, Griffith A H, Price C. P, Wright R. Liver Disease in Patients with Diabetes Mellitus. Postgraduate Medical Journal 1980; 56: 767-772.

[15] Idris A S, Mekky K F H, Abdalla B E E, Ali K A. Liver function tests in type 2 Sudanese diabetic patients. International Journal of Nutrition and Metabolism 2011; $3(2): 17-21$.

[16] Simo R, Hernadez C, Genesca J, Jardi R, Mesa J: High prevalence of hepatitis $\mathrm{C}$ virus infection in diabetic patients. Diabetes Care 1996; 19:998-1000.

[17] Jay arama N, Sudha R. A Study of Non-Alcoholic Fatty Liver Disease (NAFLD) In Type2 Diabetes Mellitus in A Tertiary Care Centre, Southern India. Journal of Clinical and Diagnostic Research doi: 3670 (published online 10th February 2012)

[18] Prashanth M, Ganesh HK, Vimal MV, John M, Bandgar T, Joshi S R, et al. Prevalence of Nonalcoholic Fatty Liver Disease in Patients with Type 2 Diabetes Mellitus. JAPI 2009; 57: 205-210. 